\title{
Correlação entre a Pressão de Perda à Manobra de Valsalva e a Pressão Máxima de Fechamento Uretral com a História Clínica em Mulheres com Incontinência Urinária de Esforço
}

\author{
Correlation of Valsalva Leak Point Pressure and Maximal Urethral Closure Pressure with \\ Clinical History in Women with Stress Urinary Incontinence
}

Paulo Cezar Feldner Jr, Leonardo Robson Pinheiro Sobreira Bezerra, Manoel João Bastista Castelo Girão Rodrigo Aquino de Castro, Marair Gracio Ferreira Sartori, Edmund Chada Baracat, Geraldo Rodrigues de Lima

\section{RESUMO}

Objetivo: analisar a relação entre a pressão de perda com manobra de Valsalva e a pressão máxima de fechamento uretral com a queixa clinica em mulheres com incontinência urinária de esforço.

Métodos: estudo retrospectivo no qual foram incluidas 164 pacientes com diagnóstico de incontinência urinária de esforço ou mista atendidas no setor de Uroginecologia e Cirurgia Vaginal do Departamento de Ginecologia da UNIFESP/EPM. As pacientes submeteram-se à anamnese padronizada, exame físico e estudo urodinâmico. A pressão de perda foi mensurada sob manobra de Valsalva (Valsalva leak point pressure - VLPP), com volume vesical de $200 \mathrm{~mL}$. O perfil uretral foi realizado utilizando-se cateter de fluxo número 8, sendo medida a pressão máxima de fechamento uretral (PMFU). As pacientes foram agrupadas conforme a queixa clinica de perda urinária aos esforços e realizou-se análise estatistica por meio do teste de $\chi^{2}$ para verificar a proporção entre as variáveis. Utilizou-se, a seguir, a análise de variância (ANOVA) para verificar diferenças entre VLPP e PMFU com relação à gravidade subjetiva da incontinência.

Resultados: a média de idade foi de 51,2 anos (19-82), sendo que 79 encontravam-se no menacme (48,2\%) e 85 (51,8\%) na pós-menopausa. A paridade média foi de 4,0 filhos (0-18). Houve correlação entre o número de pacientes com VLPP inferior a $60 \mathrm{cmH}_{2} \mathrm{O}$ e a queixa clínica ( $p<0,0001)$, sendo que o grupo com perda urinária aos mínimos esforços teve média de 69,1 $\mathrm{cmH}_{2} \mathrm{O}$ na pressão de perda, o grupo com perda urinária aos moderados esforços teve média de $84,6 \mathrm{cmH}_{2} \mathrm{O}$ e o grupo com perda urinária aos grandes esforços teve média de $90,6 \mathrm{cmH}_{2} \mathrm{O}$.

Conclusões: VLPP correlacionou-se com a queixa clínica, sendo menor no grupo com perda aos minimos esforços. Não houve correlação entre a PMFU e a queixa clínica.

PALAVRAS-CHAVE: Incontinência urinária de esforço. Manobra de Valsava. Perfil uretral. Urodinâmica. Avaliação clínica.

\section{Introdução}

A incontinência urinária de esforço é conceituada como toda perda de urina pelo óstio uretral

Universidade Federal de São Paulo - EPM / UNIFESP; Departamento de Ginecologia da Escola Paulista de Medicina, Setor de Uroginecologia e Cirurgia Vaginal Correspondência: Paulo Cezar Feldner Jr

Rua Machado Bitencourt, $\mathrm{n}^{\circ} 379$, apto 34 - Vila Clementino 04044-000 - São Paulo - SP

e-mail: pfeldner@ig.com.br externo, quando a pressão vesical excede a pressão máxima de fechamento uretral (PMFU), na ausência de atividade do músculo detrusor ${ }^{1}$. Tem causa multifatorial, sendo elemento gerador de exclusão social, interferindo na saúde física e mental da paciente e comprometendo sua qualidade de vida.

O diagnóstico da enfermidade é essencial, uma vez que a incontinência de esforço pode ser causada por deficiência esfincteriana ou por 
hipermobilidade da uretra. Sua diferenciação é prioridade antes de se propor um tratamento cirúrgico, uma vez que os procedimentos de colpossuspensão retropúbica tradicionais podem ter indices de falha de até $35 \%$ em pacientes com defeito esfincteriano ${ }^{2}$. Embora esta classificação permita distinguir duas bases fisiopatológicas distintas, é importante enfatizar que esta divisão não é absoluta. Existe um espectro no qual os dois tipos podem coexistir independentemente.

O termo "deficiência esfincteriana uretral intrinseca" refere-se a um subtipo de incontinência urinária de esforço causada pela inabilidade do mecanismo esfincteriano uretral em manter a coaptação da mucosa tanto no repouso quanto ao esforço físico ${ }^{3}$. Difere da hipermobilidade do colo vesical por fatores de risco diferentes, maior gravidade dos sintomas e pior resposta ao tratamento ${ }^{4}$.

Em geral a deficiência esfincteriana uretral intrínseca está associada a fatores de risco como a idade avançada, mielopatia congênita ou adquirida, hipoestrogenismo, cirurgias pélvicas radicais, radioterapia e cirurgias prévias para correção da incontinência urinária e/ou distopias genitais ${ }^{4}$. Do ponto de vista clínico, relaciona-se com perdas aos mínimos esforços, gravitacional ou com a mudança de posição ${ }^{5}$.

Alguns autores têm demonstrado que a pressão de perda urinária durante a manobra de Valsalva ("Valsalva leak point pressure" - VLPP) é método confiável na avaliação do mecanismo esfincteriano uretral e, portanto, no diagnóstico da deficiência esfincteriana uretral intrínseca ${ }^{6-8}$. A medida da VLPP é definida como sendo a menor pressão vesical, medida com volume conhecido, na qual se observa a perda de urina, durante aumentos da pressão abdominal ${ }^{9,10}$. Para McGuire et al. ${ }^{7}$ pacientes com incontinência urinária de esforço e VLPP inferior a $60 \mathrm{cmH}_{2} \mathrm{O}$ provavelmente terão como etiologia a deficiência esfincteriana uretral intrinseca.

Outra forma de avaliação do esfincter é feita pelo perfil pressórico uretral, em que se obtém parâmetros como a PMFU, comprimento funcional da uretra e pressão de transmissão abdominal à uretra ${ }^{1}$. Vários autores demonstraram que valores menores que $20 \mathrm{cmH}_{2} \mathrm{O}$ da $\mathrm{PMFU}$ seriam sugestivos do defeito esfincteriano ${ }^{11-13}$.

O objetivo deste estudo foi avaliar a relação entre a pressão de perda à manobra de Valsalva e a PMFU com a queixa clínica de perda aos esforços em pacientes com o diagnóstico urodinâmico de incontinência urinária de esforço.

\section{Pacientes e Métodos}

Foi feito estudo retrospectivo no qual foram incluídas 164 pacientes, atendidas no período de janeiro a dezembro de 2001 no setor de Uroginecologia e Cirurgia Vaginal da UNIFESP/EPM. Todas apresentavam o diagnóstico de incontinência urinária de esforço e foram avaliadas por anamnese, exame físico e estudo urodinâmico (fluxometria, cistometria e perfil pressórico uretral).

A anamnese consistiu em questionário padrão pelo qual se inquiriu sobre a perda de urina aos minimos, médios ou grandes esforços, bem como outros sintomas uroginecológicos (urgência miccional, urge-incontinência, disúria, hematúria, polaciúria, repleção vesical, força para iniciar a diurese, esvaziamento incompleto, enurese noturna e perda constante de urina).

As pacientes foram examinadas pela equipe de médicos do setor, em posição ginecológica e ortostática, sendo também realizada a classificação das distopias genitais existentes. Todas as pacientes submeteram-se a exame de urina (tipo I) e urocultura para descartar possiveis infecções urinárias. Utilizamos o aparelho Urosystem DS5600 de quatro canais para a realização do estudo urodinâmico. Todos os termos utilizados seguiram a padronização da Sociedade Internacional de Continência ${ }^{1}$.

O diagnóstico da incontinência urinária de esforço baseou-se na queixa clínica da paciente e na demonstração objetiva da perda urinária pela uretra por meio da manobra de Valsalva, na ausência de atividade do detrusor, durante a avaliação urodinâmica. Pacientes com evidência de instabilidade pura do detrusor não foram incluídas no estudo.

A fluxometria foi realizada nas pacientes com bexiga confortavelmente repleta utilizando-se fluxômetro com sensores de peso. Os principais parâmetros avaliados e seus respectivos padrões de normalidade foram o padrão da curva (bem definida), fluxo máximo ( $>15 \mathrm{~mL} / \mathrm{s})$, tempo de micção $(<20 \mathrm{~s})$, tempo para atingir o fluxo máximo $(<10 \mathrm{~s})$, resíduo vesical $(<50 \mathrm{~mL})$ e volume eliminado.

A cistometria foi realizada utilizando sonda uretral número 8 para infusão de água destilada, à temperatura ambiente, à velocidade de $100 \mathrm{~mL} /$ min, balão retal para medida da pressão intra-abdominal e cateter para medida da pressão intravesical. Os principais parâmetros avaliados foram o primeiro desejo miccional, a capacidade vesical máxima, a perda urinária, as contrações não inibidas e a pressão de perda. A pressão de perda foi realizada utilizando-se a manobra de Valsalva por 3 vezes consecutivas, com $200 \mathrm{~mL}$ de 
volume intravesical, considerando-se o menor valor de pressão em que a perda ocorreu. Quando não se observou perda urinária neste volume, não houve como determinar o valor do VLPP. Ressaltese, contudo, que no decorrer do exame a perda foi caracterizada e feito o diagnóstico de incontinência urinária de esforço. Os exames foram realizados na posição sentada e supina. Valores de VLPP inferiores a $60 \mathrm{cmH}_{2} \mathrm{O}$ foram considerados sugestivos de defeito esfincteriano uretral.

O perfil uretral foi realizado utilizando-se cateter de fluxo número 8 , com a bexiga repleta e em posição supina. Os parâmetros avaliados foram a PMFU e o comprimento funcional da uretra.

Utilizaram-se, inicialmente, tabelas de contingência e o teste de $\chi^{2}$ para verificar a proporção e a associação entre as variáveis pressão de perda e PMFU, baseando-se na queixa clínica da paciente de perda urinária aos esforços. Utilizaram-se, a seguir, a análise de variância (ANOVA) e o teste de comparações múltiplas de Tukey entre os grupos para verificar diferenças com relação à gravidade subjetiva da incontinência urinária. Fixou-se em 5\% o nivel de significância.

\section{Resultados}

Foram avaliadas 164 pacientes com o diagnóstico urodinâmico de incontinência urinária de esforço ou mista. A média de idade foi de 51,2 anos (19-82), sendo que 76 (47,2\%) encontravam-se no menacme e $85(52,8 \%)$ na pós-menopausa. A paridade média foi de 3,9 filhos (0-18). Em três pacientes não se obteveram os valores da pressão uretral por motivos técnicos.

A relação entre VLPP e a queixa clínica de perda urinária aos esforços está ilustrada na Tabela 1. Observamos correlação significativa $(p<0,0001)$ entre os valores da pressão de perda com o grau subjetivo da incontinência, sendo que o grupo com pressão inferior a $60 \mathrm{cmH}_{2} \mathrm{O}$ teve maior probabilidade de perda aos mínimos esforços.

Tabela 1- Distribuição das pacientes de acordo com os valores da pressão de perda e a relação com a perda urinária aos esforços.

\begin{tabular}{lcccc}
\hline \multicolumn{5}{c}{ Perda aos esforços } \\
VLPP & Mínimos & Moderados & Grandes & Total \\
\hline$<60 \mathrm{CmH}_{2} \mathrm{O}$ & 19 & 9 & 2 & 30 \\
$60-90 \mathrm{cmH}_{2} \mathrm{O}$ & 8 & 18 & 13 & 39 \\
$>90 \mathrm{CmH}_{2} \mathrm{O}$ & 10 & 16 & 12 & 38 \\
não obtido & 7 & 28 & 22 & 57 \\
\hline $\mathrm{p}<0,0001^{*}$ (teste de $\left.\chi^{2}\right)$ & & & & \\
VLPP - "Valsalva Leak Point Pressure" & &
\end{tabular}

Das 164 pacientes, conseguimos determinar o valor da VLPP em 107. Nestas utilizamos a análise de variância (ANOVA) para correlacionar os valores médios da pressão de perda conforme a queixa clínica (Tabela 2). Observamos valores menores da VLPP $\left(69,1 \mathrm{cmH}_{2} \mathrm{O}\right)$ no grupo de pacientes com queixa de perda aos mínimos esforços e 90,6 no grupo de pacientes com perda aos grandes esforços $(p<0,05)$. Quando comparadas as diferenças entre os grupos, observamos menores valores significativos neste grupo com relação ao com queixa de perda aos grandes esforços.

Tabela 2 - Correlação entre a pressão de perda (médias e erro padrão) e a intensidade do esforço.

\begin{tabular}{|c|c|c|c|}
\hline & \multicolumn{3}{|c|}{ Perda aos esforços } \\
\hline & Mínimos (G1) & Moderados (G2) & Grandes (G3) \\
\hline N & 37 & 43 & 27 \\
\hline Média $\left(\mathrm{cmH}_{2} \mathrm{O}\right)$ & 69,1 & 84,6 & 90,6 \\
\hline Erro padrão & 6,31 & 4,52 & 4,82 \\
\hline I.C. $(95 \%)$ & $56,2-81,9$ & $75,4-93,7$ & $80,7-100,5$ \\
\hline $\begin{array}{l}\text { Análise de Variância } \\
\text { Teste de comparaçõe } \\
\text { G1 x G2 (-32.58; } 1.5 \\
\text { G1 x G3 (-40.78; - } 2 \\
\text { G2 x G3 (-24.69; } 12 \\
\text { * Diferença significar }\end{array}$ & $\begin{array}{ll}\text { (ANOVA):G1 x G2 } \\
\text { es múltiplas de Tukey } \\
\text { 54) } & \mathrm{G} 1=\mathrm{G} 2 \\
\text {.25) } & \mathrm{G} 1<\mathrm{G} 3^{*} \\
.68) & \mathrm{G} 2=\mathrm{G} 3 \\
\text { nte } & \end{array}$ & $x$ G3 $p=0,02^{*}$ & \\
\hline
\end{tabular}

A relação entre PMFU e a queixa clínica de perda urinária aos esforços está ilustrada na Tabela 3. Não observamos correlação entre seus valores e o grau subjetivo da incontinência $(p=0,69)$.

Tabela 3 - Distribuição das pacientes de acordo com os valores da pressão máxima de fechamento uretral (PMFU) (menores ou maiores que $45 \mathrm{cmH}_{2} \mathrm{O}$ ) e a relação com a perda urinária aos esforços.

\begin{tabular}{lcccr}
\hline \multicolumn{5}{c}{ Perda aos esforços } \\
PMFU & Mínimos & Moderados & Grandes & Total \\
\hline$<45 \mathrm{cmH}_{2} \mathrm{O}$ & 15 & 20 & 18 & 53 \\
$>45 \mathrm{cmH}_{2} \mathrm{O}$ & 29 & 48 & 31 & 108 \\
\hline$p=0,69\left(\right.$ teste de $\left.\chi^{2}\right)$ & & &
\end{tabular}

Da mesma forma, não observamos relação entre os valores médios da PMFU conforme a queixa clínica: 50,7 para perdas aos mínimos esforços, 56,0 para as pacientes com perda aos moderados esforços e 56,8 para as pacientes com perda aos grandes esforços (Tabela 4).

\section{Discussão}

Em 1981, McGuire et al. ${ }^{9}$ foram os primeiros a introduzir o conceito de incontinência 
urinária de esforço do tipo III ou defeito esfincteriano intrinseco. Observaram que, em pacientes após falhas de cirurgias retropúbicas, a despeito da posição anatômica, a uretra não atuava como esfincter. Tais pacientes tinham deficiência no mecanismo esfincteriano caracterizado por colo vesical aberto, ao repouso, com minima ou nenhuma mobilidade sob esforço.

Tabela 4 - Relação entre a pressão máxima de fechamento uretral (média e erro padrão) com a queixa de perda urinária de esforço.

\begin{tabular}{llll}
\hline & \multicolumn{3}{c}{ Perda aos esforços } \\
& Mínimos (G1) & Moderados (G2) & Grandes (G3) \\
\hline $\mathrm{N}$ & 44 & 68 & 49 \\
Média $\left(\mathrm{cmH}_{2} \mathrm{O}\right)$ & 50,7 & 56,0 & 56,8 \\
Erro padrão & 3,15 & 2,67 & 2,79 \\
I.C. (95\%) & $44,3-57,1$ & $50,6-61,3$ & $51,2-62,4$ \\
\hline
\end{tabular}

Análise de variância (ANOVA):G1 x G2 x G3 $p=0,31$

Após esta observação, esforços têm sido feitos na definição, reconhecimento, demonstração objetiva e manejo da deficiência esfincteriana intrínseca, uma vez que constitui fator de risco para o sucesso cirúrgico ${ }^{14,15}$. Assim, a identificação pré-operatória destas pacientes é parte fundamental da propedêutica uroginecológica.

Embora parâmetros como história e exame clínico sejam são essenciais ao diagnóstico, os testes objetivos para a quantificação da função uretral são necessários para uma correta avaliação ${ }^{5}$. Não há, até o momento, padronização universal do diagnóstico do defeito esfincteriano uretral intrínseco ${ }^{16}$.

A função esfincteriana uretral é constituída por um componente ativo e outro passivo. O mecanismo passivo é composto pela musculatura lisa, posição retropúbica da uretra proximal, mucosa, plexo vascular submucoso e tecido conjuntivo periuretral. Tem como função a manutenção da pressão de fechamento e a capitação da mucosa em repouso. Já o mecanismo ativo é formado pela musculatura do esfincter uretral e do assoalho pélvico, que mantém o tônus uretral constante e contraído durante o esforço ${ }^{17}$.

Desta forma, a pressão máxima de fechamento uretral avaliaria o mecanismo passivo, informando sobre sua integridade e tônus. Já a pressão de perda mediria a capacidade do mecanismo ativo, avaliando o componente intrínseco do esfincter uretral sob esforço ${ }^{18}$.

Alguns autores demonstraram correlação entre os valores da pressão de perda e da pressão máxima de fechamento uretral ${ }^{19,20}$, ao passo que outros já não encontraram tal associação ${ }^{7,21}$.
Swift e Ostergard ${ }^{13}$ encontraram coeficiente de correlação de 0,562 entre os dois parâmetros. Identificaram uma fraca correlação clínica, uma vez que apenas $32 \%$ da variabilidade da pressão de perda foi pela variação da pressão máxima de fechamento uretral. Da mesma forma, Sultana ${ }^{19}$ encontrou resultados semelhantes ao estabelecer um coeficiente de correlação de 0,62.

Desde a sua introdução, a determinação da pressão de perda à manobra de Valsalva (VLPP) tem se mostrado clinicamente útil na avaliação do mecanismo esfincteriano e tem sido largamente utilizada como método diagnóstico ${ }^{7,22,23}$. É teste simples, realizado durante a cistometria, que avalia a interação entre a pressão vesical e a resistência uretral ${ }^{22}$. Entretanto, necessita ser padronizado com relação à espessura do cateter, volume vesical no qual a pressão é aferida e presença de distopias genitais importantes ${ }^{20,24}$.

Alguns estudos mostraram que a medida da VLPP é reprodutivel desde que o método de medida e o volume vesical sejam constantes ${ }^{8}$. Encontraram excelente correlação intra e interexaminador. Bump et al. ${ }^{21}$ relataram alta reprodutibilidade do teste em $80 \%$ de mulheres com incontinência urinária de esforço. Porém, a metodologia da medida da pressão de perda é ainda controversa. Alguns estudos demonstraram variações na determinação da pressão utilizando diferentes parâmetros, como tamanho do cateter, volume vesical, local onde a medida foi realizada, tipo de fluído e posição da paciente ${ }^{24}$.

$\mathrm{Na}$ ausência de baixa complacência vesical, uma uretra normal suporta pressões abdominais de até $250 \mathrm{cmH}_{2} \mathrm{O}$ sem que haja perda ${ }^{22}$. McGuire et al. ${ }^{7}$ relataram que $76 \%$ das mulheres com pressão de perda menor que $60 \mathrm{cmH}_{2} \mathrm{O}$ têm incontinência urinária do tipo III, avaliadas por fluoroscopia. Observaram, também, que pressões de perda acima de $90 \mathrm{cmH}_{2} \mathrm{O}$, em geral, não se relacionam com defeito esfincteriano. Concluíram que, em mulheres com incontinência urinária de esforço, a pressão abdominal necessária para provocar a perda não se relaciona com a pressão máxima de fechamento uretral.

Nitti e Combs ${ }^{18}$ demonstraram forte correlação entre a gravidade da incontinência e a medida da VLPP, em que $75 \%$ das mulheres com sintomas importantes têm pressão de perda inferior a $90 \mathrm{cmH}_{2} \mathrm{O}$. Não observaram interação entre VLPP, idade e estado menopausal.

Da mesma forma, Cummings et al. ${ }^{25}$ concluiram que a pressão de perda menor que $65 \mathrm{cmH}_{2} \mathrm{O}$ relacionou-se com grau acentuado de incontinência urinária em $77 \%$ das pacientes.

No presente estudo, observamos relação significativa entre o número de pacientes com VLPP 
inferior a $60 \mathrm{cmH}_{2} \mathrm{O}$ e a queixa clínica, denotando sua interação com a gravidade da incontinência. Da mesma forma, os valores médios da pressão de perda foram significativamente menores no grupo com perda aos mínimos esforços quando comparados ao grupo com perda aos grandes esforços.

O perfil pressórico uretral, embora utilizado por diversos autores, tem sido relatado como inadequado, especialmente quando não associado com monitorização radiográfica ${ }^{7,16,22}$. A maioria dos autores utilizam cateter eletrônico do tipo "microtip", mais sensivel que os cateteres de fluxo, embora seu uso seja limitado pelos custos e pelos artefatos. Em nosso setor, temos utilizado o perfil uretral com cateter de fluxo, sendo que valores inferiores a $45 \mathrm{cmH}_{2} \mathrm{O}$ na pressão máxima de fechamento uretral são sugestivos de provável defeito esfincteriano.

Da mesma forma que a determinação da VLPP, a avaliação da pressão uretral também pode ser afetada por vários parâmetros, como o volume vesical, posição da paciente e velocidade de infusão. Observa-se diminuição da pressão máxima de fechamento uretral com o aumento da idade. Mulheres na pós-menopausa têm valores significativamente menores quando comparadas ao menacme.

Valores da pressão uretral inferiores a 20 $\mathrm{cmH}_{2} \mathrm{O}$, utilizando "microtip", em pacientes com incontinência urinária de esforço, têm sido associados com alto risco de falhas (18-54\%) em cirurgias de colpofixação retropúbica, sendo que vários autores recomendam precedimentos do tipo "sling", injeções periuretrais ou mesmo esfincter $\operatorname{artificial}^{11}$. Contudo, outros autores não demonstraram tal relação.

Usando dados já publicados, o valor preditivo positivo da PMFU na predição do sucesso de procedimentos de colpofixação retropúbica variou entre $82-93 \%$, enquanto o valor preditivo negativo foi baixo, variando entre 4-53\%. Em outras palavras, um alto valor da pressão de fechamento uretral prediz o sucesso nestes procedimento cirúrgicos, ao passo que valores inferiores a $20 \mathrm{cmH}_{2} \mathrm{O}$ não podem ser considerados como indicadores fidedignos do diagnóstico de defeito esfincteriano e, portanto, predizer o insucesso cirúrgico ${ }^{5}$.

Segundo Swift e Ostergard $^{13}$, a pressão de perda inferior que $60 \mathrm{cmH}_{2} \mathrm{O}$ tem sensibilidade de $90 \%$, especificidade de $64 \%$, valor preditivo positivo de $56 \%$ e valor preditivo negativo de $93 \%$ no diagnóstico do perfil uretral baixo.

No presente estudo, não observamos interação entre o número de pacientes com valores da PMFU inferiores a $45 \mathrm{cmH}_{2} \mathrm{O}$ e a queixa clinica. Da mesma forma, não houve correlação entre os valores médios da PMFU nos diferentes grupos de mulheres com incontinência urinária com queixa de perda aos esforços.

A disfunção do mecanismo esfincteriano deve ser suspeitada em mulheres com incontinência urinária de esforço, principalmente quando há possibilidade de correção cirúrgica. A determinação da pressão de perda à manobra de Valsalva fornece dados para a avaliação de mulheres incontinentes de forma dinâmica e com menores custos, sendo observada, neste estudo, relação direta com a severidade da incontinência. Contudo, sua técnica necessita ser padronizada e suas variações técnicas validadas.

\section{ABSTRACT}

Purpose: to analyze the correlation between Valsalva leak point pressure and maximum urethral closure pressure and clinical symptoms in women with stress urinary incontinence. Methods: we analyzed retrospectively 164 patients with urodynamic diagnosis of stress and mixed urinary incontinence established by the Urogynecology and Vaginal Surgery Sector of UNIFESP/EPM. All patients were submmited to medical interview, physical examination and urodynamic study. Patients were divided into groups according to the subjective degree of stress urinary incontinence. Valsalva leak point pressure (VLPP) was measured with a vesical volume of $200 \mathrm{~mL}$. Urethral profile was determined using a flow catheter number 8 with measurement of maximum urethral closure pressure (MUCP). Data were compared by $\chi^{2}, A N O V A$ and Tukey tests.

Results: mean age was 51.2 years (19-82), 76 women (47.2\%) were in menacme and $85(52.8 \%)$ in postmenopausal status. Mean parity was 3.9 (0-18). The exact test for trend demonstrated a statistically significant $(p<0.0001)$ correlation between the number of patients with VLPP of 60 $\mathrm{CmH}_{2} \mathrm{O}$ or less and clinical complaints. The group with severe leakage had mean VLPP of $69.1 \mathrm{CmH}_{2} \mathrm{O}$. The group with moderated leakage had mean VLPP of $84.6 \mathrm{cmH}_{2} \mathrm{O}$ and the group with mild leakage had mean VLPP of $90.6 \mathrm{CmH}_{2} \mathrm{O}$. Conclusions: VLPP correlated with the subjective degree of stress urinary incontinence. Higher grades of stress urinary incontinence had a higher likelyhood of a low VLPP. MUCP did not correlate with clinical complaints.

KEY WORDS: Stress urinary incontinence. Valsalva maneuvre. Urethral profile. Clinical evaluation.

\section{Referências}

1. International Continence Society. Committee on Standardisation of Terminology. The Standardisation of terminology of lower urinary tract function. In: 
Ostergard DR, Bent AE, editors. Urogynecology and Urodynamics: theory and practice. $3^{\text {rd }}$ ed. Baltimore: Williams \& Wilkins; 1991. p.545-62.

2. Raz S, Sussman EM, Erickson DB, Bregg KJ, Nitti VW. The Raz bladder neck suspension: results in 206 patients. J Urol 1992; 148:845-50.

3. Urinary Incontinence Guideline Panel. Urinary incontinence in adults: clinical practice and guideline. Rockville (MD): Agency for Health Care Policy and Research, Public Health and Human Services; 1992. AHCPR Publication $n^{\circ}$ 92-0038.

4. Bump RC, Coates KW, Cundiff GW, Harris RL, Weidner AC. Diagnosing intrinsiec sphincteric deficiency: comparing urethral closure pressure, urethral axis, and Valsalva leak point pressures. Am J Obstet Gynecol 1997; 177:303-10.

5. Haab F, Zimmern PE, Leach GE. Female stress urinary incontinence due to intrinsic sphincteric deficiency: recognition and management. J Urol 1996; 156:3-17.

6. Appell RA. Collagen injection therapy for urinary incontinence. Urol Clin North Am 1994; 21:177-82.

7. McGuire EJ, Fitzpatrick CC, Wan J, et al. Clinical assessment of urethral sphincter function. J Urol 1993; 150:1452-4.

8. Gormley EA, McGuire EJ. Reproducibility of abdominal leak point pressure in the diagnosis of urinary stress incontinence. J Urol 1994; 151:478A.

9. McGuire EJ, Woodside JR, Borden TA, Weiss RM. Prognostic value of urodynamic testing in myelodysplastic patients. J Urol 1981; 126:205-9.

10.Petrou SP, Wan J. VLPP in the evaluation of female with stress urinary incontinence. Int Urogynecol J Pelv Floor Dysfunct 1999; 10:254-9.

11.Koonings PP, Bergman A, Ballard CA. Low urethral pressure and stress urinary incontinence in women: risk factor for failed retropubic surgical procedure. Urology 1990; 36:245-8.

12.Horbach NS, Ostergard DR. Predicting intrinsic urethral sphincter dysfunction in women with stress urinary incontinence. Obstet Gynecol 1994; 84:188-92.

13. Swift SE, Ostergard DR. A comparison of stress leakpoint pressure and maximal urethral closure pressure in patients with genuine stress incontinence. Obstet Gynecol 1995; 85:704-8.
14.Sand PK, Bowen LW, Panganiban R, Ostergard DR. The low pressure urethra as a factor in failed retropubic urethropexy. Obstet Gynecol 1987; 69:399-402.

15.Blaivas JG. Treatment of female incontinence secondary to urethral damage or loss. Urol Clin North Am 1991; 18:355-63.

16.Appell RA. Sphincter insufficiency: testing and treatment. Curr Opin Urol 1997; 7:197-9.

17. Ostergard DR, Bent AE. Urogynecology and urodynamics: theory and practice. $4^{\text {th }}$ ed. Baltimore: Williams \& Wilkins; 1996.

18. Nitti VW, Combs AJ. Correlation of Valsalva leak point pressure with subjective degree of stress urinary incontinence in women. J Urol 1996; 155:281-5.

19.Sultana CJ. Urethral closure pressure and leakpoint pressure in incontinent women. Obstet Gynecol 1995; 86:839-42.

20.Theofrastous JP, Bump RC, Elser DM, Wyman JF, McClish DK. Correlation of urodynamic measures of urethral resistance with clinical measures of incontinence severity in women with pure genuine stress incontinence. Am J Obstet Gynecol 1995; 173:407-14.

21.Bump RC, Elser DM, Theofrastous JP, McClish DK. Valsalva leak point pressure in women with genuine stress incontinence: reproducibility, effect of catheter caliber, and correlations with other measures of urethral resistance. Am J Obstet Gynecol 1995; 173:551-7.

22.McGuire EJ. Urodynamic evaluation of stress incontinence. Urol Clin North Am 1995; 22:551-5.

23.McGuire EJ, Cespedes RD. Proper diagnosis: a must before surgery for stress incontinence. J Endourol 1996; 10:201-5.

24.Payne CK, Raz S, Barbiaz JW. The Valsalva leak point pressure in the evaluation of stress urinary incontinence: technical aspects of measurement. J Urol 1994; 151:478A.

25.Cummings JM, Boullier JA, Parra RO, WozniakPetrofsky J. Leak point pressures in women with urinary stress incontinence: correlation with patient history. J Urol 1997; 157:818-20. 